\title{
A Perfect sTORm: The Role of the Mammalian Target of Rapamycin (mTOR) in Cerebrovascular Dysfunction of Alzheimer's Disease: A Mini-Review
}

\author{
Candice E. Van Skike Veronica Galvan \\ Department of Cellular and Integrative Physiology and The Barshop Institute for Longevity and Aging Studies, \\ University of Texas Health San Antonio, San Antonio, TX, USA
}

\section{Keywords}

Alzheimer's disease - Blood-brain barrier - Cerebral blood flow · mTOR · Neurovascular coupling · Rapamycin · Vascular density

\begin{abstract}
Cerebrovascular dysfunction is detected prior to the onset of cognitive and histopathological changes in Alzheimer's disease (AD). Increasing evidence indicates a critical role of cerebrovascular dysfunction in the initiation and progression of AD. Recent studies identified the mechanistic/mammalian target of rapamycin (mTOR) as a critical effector of cerebrovascular dysfunction in AD. mTOR has a key role in the regulation of metabolism, but some mTOR-dependent mechanisms are uniquely specific to the regulation of cerebrovascular function. These include the regulation of cerebral blood flow, blood-brain barrier integrity and maintenance, neurovascular coupling, and cerebrovascular reactivity. This article examines the available evidence for a role of $\mathrm{mTOR}$-driven cerebrovascular dysfunction in the pathogenesis of $A D$ and of vascular cognitive impairment and dementia (VCID) and highlights the therapeutic potential of targeting mTOR and/ or specific downstream effectors for vasculoprotection in AD, VCID, and other age-associated neurological diseases with cerebrovascular etiology.

(c) 2018 S. Karger AG, Basel
\end{abstract}

\section{Introduction}

Alzheimer's disease (AD) and vascular cognitive impairment and dementia (VCID) are the leading causes of dementia among the elderly, with AD and VCID amounting to approximately 60 and $20 \%$ of all dementia cases, respectively. While specific lesions and histopathological hallmarks define AD and VCID and relatively "pure" cases of $\mathrm{AD}$ and VCID can be identified in the clinic, the presentation of most $\mathrm{AD}$ and VCID cases is heterogeneous, and thus, diagnosis can present a challenge. This is because symptoms, risk factors, and etiologies of $\mathrm{AD}$ and VCID are partially overlapping and coexist in the majority of cases. Not surprisingly, pathologies common to both types of dementia are cerebrovascular in nature [1].

Brain vascular dysfunction is also involved in the etiology of related dementias, including Lewy body dementia and Parkinson disease. Indeed, cerebrovascular dysfunction is one of the earliest events in these dementias, best exemplified by diminished cerebral blood flow (CBF) [2, 3]. A recent study incorporating large datasets from the AD Neuroimaging Initiative (ADNI) suggested that vascular dysfunction indicated by decreased CBF may be the first abnormal biomarker in AD progression, as well as the one that shows the largest magnitude of change [4].

\section{KARGER}

(c) 2018 S. Karger AG, Basel

E-Mail karger@karger.com

www.karger.com/ger
Veronica Galvan

University of Texas Health San Antonio

15355 Lambda Drive

San Antonio, TX 78245 (USA)

E-Mail GalvanV@UTHSCSA.edu 
A significant barrier to effective treatments for $A D$, which are currently unavailable, is that we still do not sufficiently understand the mechanisms that drive its onset and progression. While the neuronal contributions to AD pathogenesis have been extensively studied, cerebrovascular mechanisms of $\mathrm{AD}$, which show substantial overlap with those of VCID, are only partially understood. Prominent cerebrovascular changes in AD include chronic hypoperfusion [4], increased blood-brain barrier (BBB) permeability [5], impaired neurovascular coupling [6], and diminished cerebrovascular reactivity [7].

The mechanistic/mammalian target of rapamycin (mTOR) may be a critical effector of cerebrovascular dysfunction in $\mathrm{AD}$ and potentially other dementias [8-10]. mTOR is a major signaling hub that integrates nutrient/ growth factor availability with cellular metabolism. mTOR also regulates the rate of aging across phyla, including invertebrates and mammals [11]. Rapamycin, an mTOR inhibitor, is the first drug that has been experimentally proven to slow down the rate of aging in mice [12]. Work from our lab $[8,10,13,14]$ and others [15] has identified $\mathrm{mTOR}$ as a major regulator of cerebrovascular damage and dysfunction in AD. While mTOR has a critical role in the regulation of cellular metabolism through actions at multiple signaling pathways, some mTOR-dependent mechanisms are uniquely specific to the regulation of cerebrovascular function. These include the control of CBF, BBB integrity and maintenance, neurovascular coupling, and cerebrovascular reactivity. This review will discuss the role of mTOR in the control of cerebrovascular function with a specific emphasis on dysregulation in $\mathrm{AD}$ and VCID.

\section{mTOR and CBF Deficits in AD and Other Dementias}

Chronic cerebral hypoperfusion, as evidenced by dysfunctional and/or reduced $\mathrm{CBF}$, occurs prior to cognitive impairments, brain atrophy, amyloid $\beta(\mathrm{A} \beta)$ accumulation, and a clinical diagnosis of AD [4]. Experimental evidence suggests that cerebral hypoperfusion produces cognitive impairments, synaptic alterations, and $A \beta$ oligomerization [16]. Further, primary vascular deficits lead to $A \beta$ accumulation and tau hyperphosphorylation [17]. Therefore, it has been proposed that brain microvascular dysfunction and loss of cerebrovascular density may trigger the imbalance in the levels of hyperphosphorylated tau and fibrillar $\mathrm{A} \beta$ observed in $\mathrm{AD}$, both of which are associated with a further disruption of cerebrovascu- lar function. This concept has been formulated as the "two-hit" hypothesis of AD [5].

Underlying the CBF reductions observed in $\mathrm{AD}$ are decreases in regional and global vascular density [18]. mTOR drives cerebromicrovascular density loss, leading to profound CBF deficits, by decreasing microvascular nitric oxide (NO) bioavailability in brains of mice modeling $\mathrm{AD}[8]$ through inhibition of NO synthase (NOS) activity. Therefore, mTOR attenuation with rapamycin induces endothelium-dependent cortical vasodilation via NO release [8]. In agreement with this notion, prior in vitro studies showed that mTOR inhibits endothelial NOS (eNOS) phosphorylation and activation and NOdependent arterial vasodilation [19]. Moreover, a link between the inhibition of mTOR and the activation of eNOS had been suggested by studies showing that Akt, which phosphorylates eNOS and increases NO production, can be activated by rapamycin treatment [20] and, conversely, that activation of mTOR results in Akt inhibition [20].

$\mathrm{A} \beta$, causally implicated in $\mathrm{AD}$, is generated in the brain by cleavage of the amyloid precursor protein (APP) in association with neuronal activation $[21]$. $A \beta$ is released at synaptic sites into the interstitial fluid [21]. Several physiological mechanisms act to prevent $A \beta$ accumulation, but the largest contributor is transvascular $\mathrm{A} \beta$ clearance, as over $85 \%$ of $A \beta$ is continuously cleared out of the brain through the $\mathrm{BBB}$ [5]. Disruption of transvascular $A \beta$ clearance leads to accumulation of $A \beta$ in the brain, causing $\mathrm{AD}$-like pathophysiological changes [22].

Consistent with a critical role of microvascular integrity and function in $A \beta$ removal from the brain, systemic mTOR inhibition reduces $A \beta$ levels in the brain and improves cognitive function in mouse models of AD [14], even after the onset of $\mathrm{AD}$-like deficits [8]. In these $\mathrm{AD}$ models, mTOR promotes the accumulation of $A \beta$ in the brain by inhibiting autophagy $[14,15]$ and by decreasing $\mathrm{A} \beta$ clearance as a result of decreased vascular density and reduced CBF [8]. The preservation of vascular density and vascular function that is observed as a result of mTOR attenuation in $\mathrm{AD}$ models may be sufficient to decrease brain $\mathrm{A} \beta$ and improve cognitive outcomes in $\mathrm{AD}$ and VCID. For instance, physical exercise, which restores adequate brain perfusion, reduces both $A \beta$ deposition and tau phosphorylation [23] in models of $A D$ and other tauopathies. Thus, these studies support the notion that cerebral hypoperfusion has a critical and potentially initiating role in the etiology of AD.

In agreement with a central role of mTOR-driven loss of cerebrovascular integrity and decreased cerebral perfusion in the etiology of neurological diseases of aging be- 
yond $\mathrm{AD}$, studies from our laboratory have shown that mTOR is involved in brain vascular disintegration and subsequent $\mathrm{CBF}$ deficits and cognitive impairment in low-density lipoprotein receptor knockout $\left(\mathrm{LDLR}^{-/-}\right)$ mice, a well-established model of atherosclerosis that recapitulates the vascular dysfunction of VCID [9].

\section{mTOR and BBB Breakdown in AD and Other Dementias}

The BBB is formed by a monolayer of vascular endothelial cells that line the brain microvasculature and dynamically regulate the exchange of molecules between the peripheral circulation and the central nervous system. A critical function of the $\mathrm{BBB}$ is to restrict the entry of plasma components into the brain. Clinical and experimental studies indicate that $\mathrm{BBB}$ breakdown is one of the earliest events in the pathogenesis of $\mathrm{AD}$ [5]. Further, recent studies suggest that BBB breakdown may underlie early cognitive changes in mild cognitive impairment, as evidenced by increased BBB permeability in patients with mild cognitive impairment compared to age-matched controls [24].

The BBB is maintained by intercellular tight junctions that reduce the permeability of the brain endothelium [5]. $A \beta$ induces changes in tight junction protein expression, resulting in $\mathrm{BBB}$ disruption [25]. Additionally, vascular $A \beta$ accumulation is associated with degeneration of cellular components of the vasculature, including endothelial cells, smooth muscle cells, and pericytes [26]. Although cerebral microvascular accumulation of misfolded forms of tau in $\mathrm{AD}$ and other tauopathies has been documented [27, 28], how the accumulation of misfolded tau in cerebromicrovasculature contributes to cerebrovascular dysfunction in $\mathrm{AD}$ is currently unknown. Some studies have suggested, however, that vascular tau accumulation may contribute to $\mathrm{BBB}$ breakdown in $\mathrm{AD}$ and in other tauopathies [29], suggesting that both $A \beta$ and pathogenic forms of tau contribute to $\mathrm{BBB}$ disintegration in $\mathrm{AD}$.

mTOR attenuation reduces or prevents BBB breakdown in several models of age-associated neurological disorders, suggesting a broad role of mTOR in BBB dysfunction in age-related brain disease states. MTOR inhibition with rapamycin attenuates BBB breakdown in rat models of cerebral ischemia-reperfusion injury [30,31], subarachnoid hemorrhage [32], and pharmacologically induced status epilepticus during the chronic phase [33]. These studies illustrate a key role of mTOR in BBB break-

mTOR and Cerebrovascular Dysfunction in $\mathrm{AD}$ down in several different models of age-associated neurological disease. The exact mechanisms by which mTOR promotes BBB breakdown, however, have not yet been sufficiently studied. Evidence exists that mTOR inhibition with rapamycin maintains adequate levels of tight junction protein expression in cultured cells [31]. Additionally, recent work using in vivo models suggests that BBB breakdown may follow an mTOR-dependent increase in matrix metalloproteinase 9 activity [30], which is involved in the degradation of the extracellular matrix and has been associated with various pathological processes, including cerebral hemorrhage and BBB disruption in $\mathrm{AD}$ [25]. These studies provide support for the notion that $\mathrm{mTOR}$ activity may have a critical role in $\mathrm{BBB}$ breakdown in age-associated neurological disorders, including prominently AD and VCI. Despite the abrogation of BBB breakdown, however, attenuation of mTOR activity increased cortical infarct volume in an ischemiareperfusion model [34], suggesting that mTOR activation may be necessary for neuronal survival after cerebral ischemia and reperfusion. A potential use of mTOR inhibitors preventively for stroke, or in individuals affected by diseases associated with a high risk of cerebral ischemia [34], like diabetes, might thus be limited. Because of the urgent need for interventions to slow down or block the progression of $\mathrm{AD}$ and other dementias, and the potential for the use of mTOR inhibitors in aged individuals without major undesirable side effects [35], there is a pressing need for additional research to precisely define the mechanisms of mTOR-dependent BBB disintegration and to precisely determine the functional and cognitive consequences of $\mathrm{BBB}$ restoration by $\mathrm{mTOR}$ inhibition in models of various neurological disorders. BBB breakdown may be one of the earliest events in $\mathrm{AD}$ progression [24]. Thus, understanding the mechanisms by which $\mathrm{mTOR}$ regulates $\mathrm{BBB}$ integrity may be important to devise early interventions to delay or block the development of AD.

\section{mTOR and Neurovascular Uncoupling in AD}

Rapid increases in blood flow to areas of the brain with high neuronal activity are required to maintain cellular homeostasis and function. This is accomplished through neurovascular coupling, a homeostatic response mediated by complex intercellular signaling events involving neurons, astrocytes, vascular smooth muscle cells, and endothelial cells [36]. Significant neurovascular coupling deficits are observed in patients with $\mathrm{AD}$ [6], and these 
deficits are recapitulated in several different mouse models of $\mathrm{AD}[37,38]$. NO production via activation of the neuronal form of NOS (nNOS) contributes significantly to the neurovascular coupling response by inducing local vasodilation in response to neuronal activation [6]. Dysfunctional neurovascular coupling in mouse models has been reported to occur both from reduced neuronal NO production [39] as well as from a diminished CBF response to otherwise unimpaired NO signaling [38]. Thus, differences in timing or in levels of $A \beta$ accumulation or the concomitant expression of human tau forms in different mouse models may impact neurovascular coupling and lead to cerebrovascular dysfunction through different mechanisms. Further studies are needed to better define the mechanisms underlying neurovascular coupling impairment in AD.

Since $\mathrm{mTOR}$ is a key driver of cerebrovascular damage and disintegration in several mouse models of $\operatorname{AD}[8,10]$ and in a model of VCI [9], it is reasonable to hypothesize that mTOR contributes, at least indirectly, to neurovascular coupling deficits in these models. Very little is known at present, however, about the role of mTOR in the regulation of neurovascular coupling. Interestingly, some of the mechanisms that underlie neurovascular uncoupling, including oxidative stress [40] arising from increased $\mathrm{NADPH}$-derived reactive oxygen species (ROS) production [41], are regulated by mTOR. Specifically, mTOR drives the increase in ROS observed after ischemic injury in rat brain [31]. Furthermore, ROS itself activates the Akt/ mTOR pathway in the brain [42], suggesting a feedforward mechanism involving mTOR and ROS production that leads to neurovascular uncoupling and cerebrovascular dysfunction. Indeed, a recent unbiased quantitative mass spectrometry-based proteomic study showed that proteomic alterations in the hippocampus, involving myelination, dendrite homeostasis, and oxidative stress, were associated with the upregulation of ribosomal proteins and mTOR in animals heterozygous for a null allele of tuberous sclerosis complex 1 (Tsc1 ${ }^{+/-}$mice) [43], a negative regulator of mTOR. These studies further showed that the observed proteomic changes were a direct consequence of increased mTOR activity since treatment of $\mathrm{Tscl}^{+/-}$mice with rapamycin was sufficient to normalize levels of proteins related to oxidative stress, myelin homeostasis, and protein synthesis that were altered in control-treated $\mathrm{Tscl}^{+/-}$mice. These data strongly support a direct role of mTOR in the regulation of oxidative stress in the hippocampus, as well as in the regulation of myelin homeostasis in the brain, the latter having been well established through the regulation of oligodendrocyte maturation.

\section{mTOR and Cerebrovascular Reactivity in AD}

Vasomotor reactivity, or the ability of vessels to dilate and constrict in response to physiologic or pharmacological stimuli, is mediated by coordinated responses of vascular endothelium and smooth muscle cells. Endothelium-dependent vasomotor reactivity is decreased in patients with AD and VCID [7], and smooth muscle cells degenerate in AD [44]. Most studies addressing mechanisms of vascular smooth muscle dysfunction in AD have used a mouse model of AD (Tg2576) that expresses the Swedish familial AD-mutated form of APP $\left(\mathrm{APP}_{\text {Swe }_{\mathrm{e}}}\right)$. Tg2576 mice develop extensive vascular lesions recapitulating cerebral amyloid angiopathy (CAA), the accumulation of fibrillar $A \beta$ within the cerebrovasculature. CAA is highly prevalent in $\mathrm{AD}$, but can also accumulate to a lesser extent during normal aging, and is associated with brain microhemorrhages. Tg2576 mice recapitulate the age-dependent increase in vascular fibrillar $\mathrm{A} \beta$ burden that is associated with altered vascular smooth muscle cell morphology and the loss of vascular smooth muscle cells in vascular segments with high A $\beta$ load [45]. CAA-like lesions in Tg2576 mice decrease smooth muscle cell-dependent responses necessary for relaxation and subsequent vasodilation in response to NO. These deficits are detectable prior to overt loss of vascular smooth muscle in Tg2576 mice [45]. Studies in the Swedish-Arctic APP transgenic $\left(A P P_{\text {SweArc }}\right)$ mouse model of $A D$, however, did not reveal deficits in the smooth muscle-dependent component of vasoreactivity but found significant decreases in NO bioavailability that accounted for the overall deficit in vascular reactivity documented in this model [46]. These data suggest that $A \beta$ negatively impacts several functions of different microvascular cell compartments and that specific functional consequences of $A \beta$ exposure are related to differences in timing of $A \beta$ expression and accumulation, as well as its specific localization in different transgenic mouse models of AD.

$\mathrm{AD}$ and VCID are characterized by an insensitivity to endothelium-dependent vasodilation, and these deficits are causally linked to decreased vascular reactivity in $\mathrm{AD}$. The endothelium-dependent component of brain microvascular dilation depends on the synthesis of NO by eNOS within brain microvascular endothelial cells. In agreement with a critical role of brain vascular endothelial dysfunction in the cerebrovascular deficits of $\mathrm{AD}$, changes in eNOS activity have been linked to AD pathology. Accumulation of both $A \beta$ and neurofibrillary tangles is associated with reduced eNOS expression in the brain 
capillaries of human patients with $\mathrm{AD}$ and with endothelial cell apoptosis [47]. Loss of eNOS activity can in turn promote tau phosphorylation in a mouse model of $\mathrm{AD}$ [48], suggesting that the accumulation of $\mathrm{AD}$ pathology and the dysregulation of eNOS may be linked in a feedforward loop. However, the mTOR inhibitor rapamycin induces eNOS phosphorylation, restores endotheliumdependent vasodilation, and produces NOS-dependent restoration of baseline CBF [8].

In agreement with a central role of cerebrovascular dysfunction in the early stages of $\mathrm{AD}$ progression, young adult carriers of the ApoE4 allele show reduced cerebrovascular reactivity before developing detectable cognitive impairments [49]. Interestingly, mTOR attenuation is sufficient to preserve cerebrovascular integrity and function in ApoE4 transgenic mice [10], suggesting that mTOR is a critical driver of early cerebrovascular dysfunction in a model of sporadic AD associated with ApoE4 carrier status. Since mTOR activity reduces NOSdependent cerebromicrovascular NO bioavailability, mTOR inhibitors may be efficacious for the preservation of cerebrovascular function as an early treatment and intervention in $\mathrm{AD}$.

Intriguing recent studies (reviewed in [50]) have suggested that overactivation of the renin-angiotensin system may underlie cerebrovascular dysfunction in $\mathrm{AD}$. Angiotensin (Ang)-II and Ang-III levels are higher in $\mathrm{AD}$ patients compared to age-matched control subjects, and Ang-III is strongly associated with both $A \beta$ and phosphorylated tau pathology [51]. In agreement with an important role of the renin-angiotensin axis in $\mathrm{AD}$ pathogenesis, it has been reported that centrally acting angiotensin-converting enzyme inhibitors temporarily delay progression of $\mathrm{AD}$ cognitive deficits [52], and recent studies in mouse models of AD showed that the beneficial effects of the angiotensin receptor blocker losartan on cognitive performance and cerebrovascular function may be related to its selective blockage of the Ang-IV receptor [53]. Furthermore, overactivation of Ang-II has been shown to contribute to cerebrovascular pathogenesis in stroke through vasoconstriction, activation of proinflammatory factors, and increased oxidative stress in the parenchyma [54]. Ang-II strongly activates mTOR, and blockade of its receptor, the angiotensin type I receptor, reduces activity of the mTOR pathway [55], which can ameliorate cerebral microcirculatory changes to improve brain perfusion [56]. Additionally, it was shown that mTOR inhibition with rapamycin prevented in vitro aortic endothelial cell dysfunction induced by Ang-II, including preservation of eNOS phosphorylation, NO pro-

mTOR and Cerebrovascular Dysfunction in $\mathrm{AD}$ duction, and vasodilation [57]. Taken together, these data suggest that improvements in cerebrovascular function from therapeutic interventions targeting the reninangiotensin system in $\mathrm{AD}$ may at least partially result from the direct inhibition or downregulation of mTOR activity.

\section{Conclusions and Perspectives}

Recent studies highlight the role of cerebrovascular dysfunction in the pathogenesis of AD and VCID. Given that cerebrovascular dysfunction can be detected prior to the onset of cognitive impairments, presentation of $\mathrm{AD}$ associated pathologies, and a diagnosis of $\mathrm{AD}$ [4], there has been recent interest in exploring the potential of $\mathrm{CBF}$ deficits as a noninvasive biomarker for risk of $\mathrm{AD}$ development, as well as a target for intervention early in the pathogenesis of $\mathrm{AD}$. Our laboratory and others have identified the mTOR pathway as a potential target for brain vasculoprotection in AD and VCID [8-10, 14]. Since mTOR-dependent cerebrovascular dysfunction is not limited to AD models, the mTOR pathway may be a suitable target for early intervention in several different disorders beyond $\mathrm{AD}$ that have cerebrovascular dysfunction as a common etiology.

Adverse effects of rapalogs at doses used in oncology or organ transplantation may be of concern [58]. Thus, it is crucial that the mechanisms of rapamycin- and rapalog-induced neuroprotection and vasculoprotection are elucidated to enable the design of better strategies, such as the use of existing drugs, or development of new ones, that target key effectors of rapamycin-induced neuroprotection and/or vasculoprotection while avoiding potential undesirable side effects. However, a relatively recent study showed that a 6-week course of rapamycin enhanced the response to the influenza vaccine by about $20 \%$ in elderly volunteers over 65 years of age, without significant adverse events [35]. These findings underscore the need for additional exploratory proof-of-concept studies in the elderly, which would be the target population for interventions aimed at blocking or delaying $\mathrm{AD}$ progression early during development of the disease. Since mTOR inhibitors are available clinically, translational studies of dementias with cerebrovascular dysfunction could follow quickly. As these studies are performed, rapamycin- or rapalog-based therapies to treat $\mathrm{AD}$ can be designed that take advantage of strategies such as intermittent administration, as well as personalized dosage and frequency of treatment. 


\section{Acknowledgements}

These authors are supported by Merit Review Award I01 BX002211-01A2 from the United States Department of Veterans Affairs, Biomedical Laboratory Research and Development Service, a William \& Ella Owens Medical Research Foundation Grant, and a NIH Institute for Integration of Medicine and Science Award to V.G. C.E.V.S. was funded by Alzheimer's Association AARF17-504221 and NIA T32AG021890. We further acknowledge the support of the San Antonio Nathan Shock Center of Excellence in the Biology of Aging (2 P30 AG013319-21), the San Antonio Medical Foundation, the JMR Barker Foundation, and the generous support from the Robert L. Bailey and daughter Lisa K. Bailey Alzheimer's Fund in memory of Jo Nell Bailey.

\section{Disclosure Statement}

The authors have no conflicts of interest to report.

\section{References}

1 Trigiani LJ, Hamel E: An endothelial link between the benefits of physical exercise in dementia. J Cereb Blood Flow Metab 2017;37: 2649-2664.

2 Binnewijzend MA, et al: Distinct perfusion patterns in Alzheimer's disease, frontotemporal dementia and dementia with Lewy bodies. Eur Radiol 2014;24:2326-2333.

-3 Syrimi ZJ, et al: Arterial spin labelling detects posterior cortical hypoperfusion in non-demented patients with Parkinson's disease. J Neural Transm (Vienna) 2017;124:551-557.

4 Iturria-Medina Y, et al: Early role of vascular dysregulation on late-onset Alzheimer's disease based on multifactorial data-driven analysis. Nat Commun 2016;7:11934.

5 Zlokovic BV: Neurovascular pathways to neurodegeneration in Alzheimer's disease and other disorders. Nat Rev Neurosci 2011; 12:723-738.

6 Girouard H, Iadecola C: Neurovascular coupling in the normal brain and in hypertension, stroke, and Alzheimer disease. J Appl Physiol (1985) 2006;100:328-335.

7 Vicenzini E, et al: Cerebrovascular reactivity in degenerative and vascular dementia: a transcranial Doppler study. Eur Neurol 2007; 58:84-89.

8 Lin $\mathrm{AL}$, et al: Chronic rapamycin restores brain vascular integrity and function through NO synthase activation and improves memory in symptomatic mice modeling Alzheimer's disease. J Cereb Blood Flow Metab 2013;33:1412-1421.

9 Jahrling JB, et al: mTOR drives cerebral blood flow and memory deficits in $\mathrm{LDLR}^{-/-}$mice modeling atherosclerosis and vascular cognitive impairment. J Cereb Blood Flow Metab 2017, DOI: $10.1177 / 0271678 X 17705973$.

10 Lin AL, et al: Rapamycin rescues vascular, metabolic and learning deficits in apolipoprotein $\mathrm{E} 4$ transgenic mice with pre-symptomatic Alzheimer's disease. J Cereb Blood Flow Metab 2017;37:217-226.

11 Bitto A, et al: Biochemical genetic pathways that modulate aging in multiple species. Cold Spring Harb Perspect Med 2015;5.

$\checkmark 12$ Harrison DE, et al: Rapamycin fed late in life extends lifespan in genetically heterogeneous mice. Nature 2009;460:392-395.
13 Pierce A, et al: Overexpression of heat shock factor 1 phenocopies the effect of chronic inhibition of TOR by rapamycin and is sufficient to ameliorate Alzheimer's-like deficits in mice modeling the disease. J Neurochem 2013;124:880-893.

14 Spilman P, et al: Inhibition of mTOR by rapamycin abolishes cognitive deficits and reduces amyloid-beta levels in a mouse model of Alzheimer's disease. PLoS One 2010; 5:e9979.

15 Caccamo A, et al: Genetic reduction of mammalian target of rapamycin ameliorates Alzheimer's disease-like cognitive and pathological deficits by restoring hippocampal gene expression signature. J Neurosci 2014;34: 7988-7998.

16 Wang X, et al: Cerebrovascular hypoperfusion induces spatial memory impairment, synaptic changes, and amyloid-beta oligomerization in rats. J Alzheimers Dis 2010;21: 813-822.

17 Schreiber S, et al: Interplay between age, cerebral small vessel disease, parenchymal amyloid-beta, and tau pathology: longitudinal studies in hypertensive stroke-prone rats. J Alzheimers Dis 2014;42(suppl 3):S205-S215.

$\checkmark 18$ Fischer VW, Siddiqi A, Yusufaly Y: Altered angioarchitecture in selected areas of brains with Alzheimer's disease. Acta Neuropathol 1990;79:672-679.

19 Cheng C, et al: Rapamycin modulates the eNOS versus shear stress relationship. Cardiovasc Res 2008;78:123-129.

20 Sun SY, et al: Activation of Akt and eIF4E survival pathways by rapamycin-mediated mammalian target of rapamycin inhibition. Cancer Res 2005;65:7052-7058.

21 Kamenetz F, et al: APP processing and synaptic function. Neuron 2003;37:925-937.

22 Ramanathan A, et al: Impaired vascular-mediated clearance of brain amyloid beta in Alzheimer's disease: the role, regulation and restoration of LRP1. Front Aging Neurosci 2015;7:136.

23 Liu HL, et al: Long-term treadmill exercise inhibits the progression of Alzheimer's diseaselike neuropathology in the hippocampus of APP/PS1 transgenic mice. Behav Brain Res 2013;256:261-272.
24 Montagne A, et al: Blood-brain barrier breakdown in the aging human hippocampus. Neuron 2015;85:296-302.

25 Brkic M, et al: Amyloid beta oligomers disrupt blood-CSF barrier integrity by activating matrix metalloproteinases. J Neurosci 2015;35: 12766-12778.

26 Erickson MA, Banks WA: Blood-brain barrier dysfunction as a cause and consequence of Alzheimer's disease. J Cereb Blood Flow Metab 2013;33:1500-1513.

27 Castillo-Carranza DL, et al: Cerebral microvascular accumulation of tau oligomers in Alzheimer's disease and related tauopathies. Aging Dis 2017;8:257-266.

28 Vidal R, et al: Senile dementia associated with amyloid beta protein angiopathy and tau perivascular pathology but not neuritic plaques in patients homozygous for the APOE- $\varepsilon 4$ allele. Acta Neuropathol 2000;100:1-12.

29 Blair LJ, et al: Tau depletion prevents progressive blood-brain barrier damage in a mouse model of tauopathy. Acta Neuropathol Commun 2015;3:8.

30 Guo W, et al: Rapamycin alleviates brain edema after focal cerebral ischemia reperfusion in rats. Immunopharmacol Immunotoxicol 2014;36:211-223.

31 Li H, et al: Evaluation of the protective potential of brain microvascular endothelial cell autophagy on blood-brain barrier integrity during experimental cerebral ischemia-reperfusion injury. Transl Stroke Res 2014;5:618-626.

32 You W, et al: Inhibition of mammalian target of rapamycin attenuates early brain injury through modulating microglial polarization after experimental subarachnoid hemorrhage in rats. J Neurol Sci 2016;367:224-231.

33 van Vliet EA, et al: Blood-brain barrier leakage after status epilepticus in rapamycintreated rats I: magnetic resonance imaging. Epilepsia 2016;57:59-69.

$34 \mathrm{Chi} \mathrm{OZ}$, et al: Effects of rapamycin pretreatment on blood-brain barrier disruption in cerebral ischemia-reperfusion. Neurosci Lett 2016;620:132-136.

35 Mannick JB, et al: mTOR inhibition improves immune function in the elderly. Sci Trans Med 2014;6:268ra179. 
36 Tarantini S, et al: Impaired neurovascular coupling in aging and Alzheimer's disease: contribution of astrocyte dysfunction and endothelial impairment to cognitive decline. Exp Gerontol 2017;94:52-58.

37 Tarantini S, et al: Demonstration of impaired neurovascular coupling responses in TG2576 mouse model of Alzheimer's disease using functional laser speckle contrast imaging. Geroscience 2017, Epub ahead of print.

-38 Lourenco CF, et al: Neurovascular uncoupling in the triple transgenic model of $\mathrm{Alz}$ heimer's disease: impaired cerebral blood flow response to neuronal-derived nitric oxide signaling. Exp Neurol 2017;291:36-43.

$>39$ Rancillac A, Geoffroy H, Rossier J: Impaired neurovascular coupling in the APPxPS1 mouse model of Alzheimer's disease. Curr Alzheimer Res 2012;9:1221-1230.

40 Hamel E, et al: Oxidative stress and cerebrovascular dysfunction in mouse models of Alzheimer's disease. Exp Physiol 2008;93: 116-120.

41 Park L, et al: NADPH-oxidase-derived reactive oxygen species mediate the cerebrovascular dysfunction induced by the amyloid beta peptide. J Neurosci 2005;25:1769-1777.

$\checkmark 42$ Chen S, et al: N-acetyl-L-cysteine protects against cadmium-induced neuronal apoptosis by inhibiting ROS-dependent activation of Akt/mTOR pathway in mouse brain. Neuropathol Appl Neurobiol 2014;40:759-777.
43 Wesseling H, Elgersma Y, Bahn S: A brain proteomic investigation of rapamycin effects in the $\mathrm{Tscl}^{+/-}$mouse model. Mol Autism 2017;8:41.

-44 Farkas E, Luiten PG: Cerebral microvascular pathology in aging and Alzheimer's disease. Prog Neurobiol 2001;64:575-611.

45 Christie R, et al: Structural and functional disruption of vascular smooth muscle cells in a transgenic mouse model of amyloid angiopathy. Am J Pathol 2001;158:1065-1071.

46 Merlini M, et al: Reduced nitric oxide bioavailability mediates cerebroarterial dysfunction independent of cerebral amyloid angiopathy in a mouse model of Alzheimer's disease. Am J Physiol Heart Circ Physiol 2017; 312:H232-H238.

47 Fossati S, et al: Differential activation of mitochondrial apoptotic pathways by vasculotropic amyloid-beta variants in cells composing the cerebral vessel walls. FASEB J 2010;24: 229-241.

48 Austin SA, Katusic ZS: Loss of endothelial nitric oxide synthase promotes p25 generation and tau phosphorylation in a murine model of Alzheimer's disease. Circ Res 2016;119: 1128-1134.

49 Suri S, et al: Reduced cerebrovascular reactivity in young adults carrying the APOE $\varepsilon 4$ allele. Alzheimers Dement 2015;11:648-657.e1.

50 Kehoe PG, Miners S, Love S: Angiotensins in Alzheimer's disease - friend or foe? Trends Neurosci 2009;32:619-628.

51 Kehoe PG, et al: Angiotensin-III is increased in Alzheimer's disease in association with amyloid-beta and tau pathology. J Alzheimers Dis 2017;58:203-214.
52 Fazal K, et al: Associations of centrally acting ACE inhibitors with cognitive decline and survival in Alzheimer's disease. BJPsych Open 2017;3:158-164.

53 Royea J, et al: Angiotensin IV receptors mediate the cognitive and cerebrovascular benefits of losartan in a mouse model of Alzheimer's disease. J Neurosci 2017;37:5562-5573.

54 Arroja MMC, Reid E, McCabe C: Therapeutic potential of the renin angiotensin system in ischaemic stroke. Exp Transl Stroke Med 2016;8:8.

55 Diniz GP, Carneiro-Ramos MS, BarretoChaves ML: Angiotensin type 1 receptor mediates thyroid hormone-induced cardiomyocyte hypertrophy through the Akt/GSK-3beta/mTOR signaling pathway. Basic Res Cardiol 2009;104:653-667.

56 Estato V, et al: Blockade of the renin-angiotensin system improves cerebral microcirculatory perfusion in diabetic hypertensive rats. Microvasc Res 2013;87:41-49.

57 Kim JA, Jang HJ, Martinez-Lemus LA, Sowers JR: Activation of mTOR/p70S6 kinase by ANG II inhibits insulin-stimulated endothelial nitric oxide synthase and vasodilation. Am J Physiol Endocrinol Metab 2012; 302:E201-E208.

58 Arriola Apelo SI, Lamming DW: Rapamycin: an inhibiTOR of aging emerges from the soil of Easter Island. J Gerontol A Biol Sci Med Sci 2016;71:841-849. 